\title{
Readability and Academic Communication: A Comparative Study of Undergraduate Students' and Handbook of Three Ghanaian Universities
}

\author{
William Kodom Gyasi \\ Department of Communication Studies University of Cape Coast, Ghana
}

\begin{abstract}
Essentially, this study investigated the readability of handbooks of three reputable universities in Ghana, namely: University of Ghana, Kwame Nkrumah University of Science and Technology and University of Cape Coast. The study conducted an in-depth investigation into the readability of these handbooks, using seven readability indexes. These readability indexes include: Flesch-Kincaid Grade Level, Flesch Reading Ease, Gunning Fog Index, Coleman-Liau Index, SMOG Index, Automated Readability Index and Lisear Write Formula. The readability consensus for the 7 readability indexes showed that these handbooks are very difficult to comprehend when measured in terms of readability indexes and that they were generally written to be understood by university graduates and in some cases even above the reading level of university graduates. The study also established that there are no statistically significant differences across the mean scores of the readability of the three handbooks. It is therefore recommended that the hallmark of texts contained in students' handbooks should be sensitive to students' reading level. It is belief that this can be achieved through the use of plain language in writing students' handbooks.
\end{abstract}

Keywords: text readability; readability index, Flesch-Kincaid Grade Level, Flesch Reading Ease, Gunning Fog, Coleman-Liau Index, SMOG Index, Automated Readability Index, Lisear Write Formula and Ghanaian Universities Students' Handbook.

\section{Introduction}

As streetlight lights up the roadway in the night and a map provides guidance and direction to keep a traveller from straying, so is the university students' handbook (Matz, 2012). According to Webster's Collegiate Dictionary ( ${ }^{\text {th }}$ edition), handbook is a concise reference book covering a particular subject. On the other hand, Oxford Online Dictionaries describes it as a book giving information such as facts on a particular subject or instructions on a variety of topics, ranging from private to public matters. Saxonis, a Medieval Latin Dictionary sees it as vade mecum (Latin, "go with me") or pocket reference that is intended to be carried at all times. It may also be referred to as an enchiridion (Late Latin term for a small manual or handbook).

There is no gainsaying that student's handbook is fundamental to an enjoyable and rewarding university education. While it is not a formal contract or agreement, the handbook is usually designed to assist students navigate, familiarize and acclimatize themselves with the university environment and live up to the institution's expectations and requirements (Pannekoek, 2012). Handbooks are a source of ready reference for school policies, procedures, services and other relevant information geared towards a fruitful course of study at the university (Drucker, 2012). For example, on page 11 of the handbook of the oldest and largest of the Ghanaian universities and tertiary institutions, the university of Ghana (founded in 1948), detailed information is provided in connection with the location of the institution. It is stated that the campus of the university lies about 13 kilometers north-east of Accra, the capital of Ghana, at an altitude of between 90 and 100 metres.

From the main university gate on the Dodowa Road, the University Avenue extends to Commonwealth Hall on Legon Hill. Providing detailed information on regulations for the conduct of university examination, the handbook of Kwame Nkrumah University of Science and Technology stipulates that all students, both males and females, are entreated to dress decently to the examination Hall. It further states that any candidate who does not dress decently would be refused entry into the Examination Hall. The University of Cape Coast Students' handbook on page 34 under the subheading, "Discipline" states that the University of Cape Coast is a community of Students, Lecturers and Administrators, hence it is required that as members of this diverse community each one should live by standards of proper conduct so that one member's freedom will not impinge on another member's right. In all cases of breach of discipline, punishment may involve fines, suspension or dismissal.

From the foregoing, it is clear that information contained in university handbook is invaluable and a pre-requisite to successful and rewarding university education. How can a student perform well if they don't understand the policies and regulations guiding examination, lecture theatres, cohabitation in the halls and other activities on campus? Emphasizing the important place of students' handbook in students academic life, Farmer et al. (2007) affirms that handbook constitute the most fundamental source of reference for students. Such 
educational and instructional material comprise rich texts which have profound influence on the cognitive and perceptive capabilities of students when written to commensurate with students age and knowledge levels, prepared on the basis on which education programs are grounded so as to transfer the contained knowledge to students. In line with this statement, Unsal and Gunes (2008) stated that handbooks are important tools enabling students to work independently; being fully aware of what is required of them.

In order to aid understanding and comprehensibility of university students' handbook, authors of this document must ensure that it is written at a level that commensurate with students' reading ability. This should be of serious concern to university administration, because, Wellington and Osborne (2001) indicated that language use is the major barrier to most students in the learning process. Since English is the official language of Ghana, it is the medium of both verbal and non-verbal instruction in the university. This medium of instruction and its influence on learning has become an important issue (Yong, 2010). Lemke (1997) asserts that students encounter numerous problems due to learning English as a foreign language, of which they seldom use at home and other social settings. Several researchers have indicated that the language used in some instructional material exceeds the normal experience of many university students (Lynch, et al., 1972 cited in Letsoalo, 1996, p. 184) for whom they are written. Curtis and Millar (1988) argued that if the understanding of handbook language is difficult for English speakers, it is likely to be even more difficult for students who speak and read English as a second language. In the Ghanaian context, it has long been recognized that students encounter enormous problems reading and comprehending English as a second language (Heppner et al., 1997; Yong, 2003, Mohiddin, 2007; Romaizah, 2009 ;).

It is against this background that the present study will carry out an extensive comparison of the readability of students' handbook of three universities in Ghana: University of Ghana, Kwame Nkrumah University of Science and Technology, and University of Cape Coast by means of seven popular readability indexes: Flesch-Kincaid Grade Level, Flesch Reading Ease, Gunning Fog Index, Coleman-Liau Index, SMOG Index, Automated Readability Index and Lisear Write Formula.

\section{Statement of the Problem}

Studies have shown that truancy, examination malpractices, and poor performance persists despite guide and direction provided in students' handbook, suggesting that information contained in the handbook is not really influencing students' activities on university campuses (Atabong et al., 2010; Fordjour et. al., 2011; Bediako, 2013). In his book, Ghanaian English: An exploratory survey, Sey (1973:7) posits that in connection with language use and style, educated and learned Ghanaians have the tendency of using learned and archaic forms coupled with flamboyance of prose style and frequent cases of hyper-correctness. Little wonder public speakers, journalists and writers who are known for this flamboyant prose style and the use of learned forms in Ghana are those ascribed linguistic excellence.

As a result of the above stated tendency among writers in Ghana, several handbooks maybe produced without due consideration for its readability or whether the intended audience can comprehend its content. Since readability indexes measure comprehensibility of written text and comprehensibility is sine qua non to acting in harmony with what is read and understood, it would be pleasing to examine the readability of students' handbook of universities in Ghana. In doing this, the researcher uses the handbooks of University of Ghana, Kwame Nkrumah University of Science and Technology and University of Cape Coast for the study. The choice of these universities is as a result of the fact that they are generally perceived to be the three leading universities in Ghana. Also, the choice is as a result of the longstanding reputation of these three universities as icon of excellence. These universities also constitute a fair representation of universities in Ghana.

This study was intended to:

\section{Objectives of the study}

i. Establish the readability of Ghanaian universities students' handbook measured in terms of readability indexes.

ii. Ascertain if there is any significant difference between the readability of Ghanaian universities students' handbooks.

iii. Find out if there are variations in the scores of readability indexes.

IV. Research Questions

i. How readable are Ghanaian universities students' handbook measured in terms of readability indexes?

ii. Are there variations in the scores of readability indexes?

iii. Are there any significant differences in the readability of Ghanaian universities students' handbook? 


\section{Hypothesis}

Ghanaian universities students' handbooks are difficult to comprehend when measured in terms of readability indexes.

\section{Literature Review And Theoritical Perspective}

This section of the research has two parts, the first part focuses on the theory that underpins the study. Here, attention is given to social constructionism, a theory attributed to Vygotsky (1978). The second part of this section discusses readability indexes and reviews of some works done in the area. Attention is also given to the place of Ghana in World Englishes, drawing attention to the need for English Language, which has become the soul medium of instruction in secondary and tertiary institutions to be handled in a manner that will enhance academic communication.

\section{i. Social Constructionism}

Social constructionism as a theory, attributed to Vygotsky (1978), the theory refers to the development of phenomena relative to social contexts. According to Flamand (1999), the theory accounts for the ways in which phenomena are socially constructed. Social construction theory thus focuses on the ways we think about our experience and analysis of the world. Hackling (1999), also asserts that a major focus of social constructionism is to uncover the ways individuals and groups participate in the construction of their perceived social reality. It involves looking at the ways social phenomena are created, institutionalized, known and made into tradition by humans. He underscores that the social construction of reality is an ongoing, dynamic process that is (and must be) reproduced by people acting on their interpretation and their knowledge of it.

Social constructionism is associated with what is called social construct. Boghossian (2001) and Flamand (1999), underscore that social construct is anything that exists as a product of human social interaction instead of objective, human independent existence. It can be said that social constructionism is a concept or practice; that is, the creation of a particular group. Social constructs are generally understood to be the by-products of countless human choices rather than laws resulting from divine will or nature. Gergen (1985) had explained social constructionism: Social constructionist inquiry is principally concerned with explicating the process by which people come together to describe, explain, or otherwise account for the world (including themselves) in which they live. It attempts to articulate common forms of understanding as they now exist, as they have existed in prior historical periods, and as they might exist should creative attention be so directed (p. 266). The constructionist attempts to ascertain how people explain their existence as a group through what pertains now, was and will be if conscious effort are taken to enable the common forms of doing things in that group. Relating the theory of social constructionism to writing, writing is essentially a social act, in which the writer must construct meaning in a manner conventional in a particular community. This school of thought ascribes to the learner-writer a high level of awareness of its central tenant (be it cognitive strategies in the writing process, or writing as a social practice). It portrays the experience of writing from the point of view of awareness - either of the cognitive process/problem solving, or of social action. The view that knowledge is created through the discourses of social communities has its origin in the theory of social constructionism. Thus the ways we comprehend the world, the categories and concepts we use, are not 'truths' proven and fixed for all time but are specific to particular cultures and periods. In other words, our knowledge does not result from objective descriptions of what the world is really like, but emerges in part through our perceptions of that world during our interactions. No matter how careful our experiments or rigorous our armchair reasoning, they always involve interpretation, and interpretation always depends, at least in part, on the assumptions researchers bring to the problem they are studying. More simply, knowledge is 'the social justification of belief', and in academic contexts, this justification is accomplished through academic discourses (Rorty, 1979. p. 170). In sum, academics cannot step outside the beliefs of their social groups to tell us 'what the world is really like' but they have to draw on conventional ways of producing agreement.

Social constructionism focuses on the creation of meaning, on the existence, the development and the role of joint meaning (Katerm et al., 2004).

Since the theory of social constructionism dwells on the ways in which individuals and groups participate in the creation of their perceived social reality, it is found appropriate for the present research. Thus the way language is used in the writing of Ghanaian university students' handbooks resulting in the level of difficulty of the handbooks can be seen from the point of view the universities individually participating in the creation of realities of themselves to the outside world and even to members of the university community, in the practice of handbook writing, is what makes the theory to be useful in this present study. Indeed, Hyland (1999) asserts that academic knowledge is now perceived as a social accomplishment, the outcome of a cultural activity shaped by ideology and constituted by agreement between the writer and a potentially skeptical discourse community. Academic writing does not exist in vacuum; it is situated in a disciplinary community for it to be accepted as one of its kind. 


\section{ii. Discussion of Readability Indexes}

Lively and Pressey (1923) were among the pioneers of readability formulas. In all, researchers have proposed more than 200 readability formulas (Klare, 1984). Almost without exception, readability formulas are based on syntactic and semantic complexity. Basically, the number of words per sentence determines syntactic complexity. The basis for measuring semantic complexity is either by word familiarity as defined by its inclusion on a word list or the number of syllables per word.

According to Fry (2002), readability of classroom materials or public documents, usually refers to a numerical or grade-level score that is obtained by applying a mathematical formula to a sample of text. The classic readability formulas predict comprehension (McLaughlin, 1974). Most do so by providing a numerical score representing the educational level necessary to read a document with ranges of $50 \%$ to $75 \%$ comprehension. However, Klare (1974) indicated that the SMOG formula predicts $100 \%$ comprehension. In connection with popular readability indexes, Burke and Greenberg (2010) intimated that the mathematical formula used to determine the difficulty of a given text typically takes into consideration issues such as sentence complexity (measured by sentence length) and vocabulary difficulty (measured by either the number of letters or syllables in words or by a comparison to lists of easy or difficult words). In effect, readability formulas measure the relationship between the difficulty experienced when reading a text and the linguistic features, specifically word meaning and sentence structure, of that text (McLaughlin, 1969) on the web and also the one found in a word processing program. Burke and Greenberg's (2010) findings also revealed that the SMOG readability scores for books written above the fourth-grade level were consistently high while Flesch-Kincaid scores for the same books were consistently low. Based on their finding, Burke and Greenberg (2010) summarized the comparison between the readability indexes as shown in Table 1 and recommended the use of two or more readability indexes to ascertain the readability of a given text, textbook or handbook in view of the varying scores obtainable from readability indexes.

Table 1: Comparison of Some Readability Indexes

\begin{tabular}{ll}
\hline Selection Criterion & Readability tool \\
\hline $100 \%$ comprehension & Always use SMOG \\
Material below fourth grade & Use Fry \\
Identify difficult words & Always use Dale-Chall \\
Easiest & Flesch-Kincaid Grade level - Can do straight \\
& from Microsoft Word \\
Cautions & Flesch-Kincaid often scores lower than other \\
& measures other \\
& SMOG often scores higher than ot \\
& measures
\end{tabular}

Source: Burke and Greenberg (2010)

According Brutt-Griffler (2002), World Englishes has been described as a phase in the history of the English language. This phase has witnessed the transformation of English from the mother tongue of a handful of nations to a language being used by far more speakers in non-mother tongue settings. The changes that have accompanied this spread-the multiplicity of varieties--result not from the faulty and imperfect learning of the non-mother tongue speakers, but from the nature of the process of micro acquisition, language spread and change.

Due to the transformation the English language is presently undergoing, world English has produced certain varieties. Among these varieties, the term, 'New Englishes' is used to describe regional and national varieties of the English language used in places, such as Ghana, where it is not the mother-tongue of the majority of the population (Nordquist, 2013). Mollin (2006) indicated that Kachru coined the term non-native Englishes to refer to the variety of English which is not a native variety. This term has gained popularity that Platt, Weber and Ho (1984) designate an English variety with the following characteristics as non-native English:

i. It has developed through the education system (possibly even as a medium of education at a certain level), rather than as a first language of the home.

ii. It has developed in an area where a native variety of English was not spoken by a majority of the population.

iii. It is used for a range of functions (for example, letter-writing, government communications, literature, as a lingua franca within a country and in formal contexts).

iv. It has become nativised, by developing a subset of rules which mark it as different from American or British English.

Jenkins (2009) affirms that Kachru used his three-concentric model of world Englishes to represents the way in which English had been spread, the way people acquired the language, and the use of English. In 
essence, Kachru's concentric model is built on the historical context of English, the status of the language, its geographical distribution and its functions in various regions.

\section{Kachru's Three Concentric Model of World Englishes}

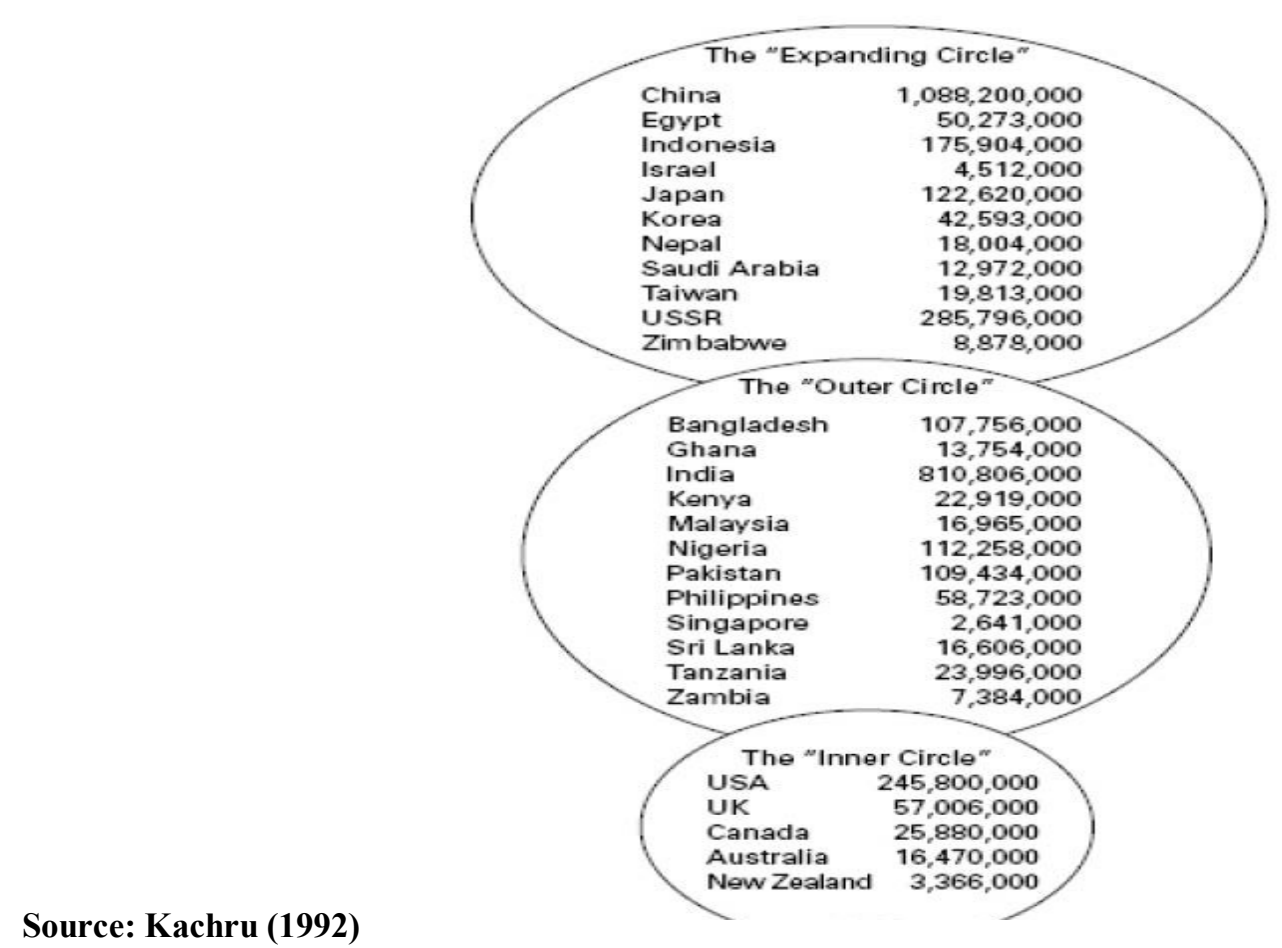

\section{iii. Classification of World Englishes}

As depicted in the above diagram, Kachru proposes that the countries where English has traditionally been the native language, would belong to the Inner Circle; hence, they would be the "norm-providing". Countries where English has official status ("norm-developing") would be located in the Outer circle, of which Ghana is inclusive. And, countries where English has no official status would be in the Expanding circle; thus, they would be "norm-dependent".

However, irrespective of the wide publicity and acceptance of this model, it has some weaknesses in terms of uses and users of English, Jenkins (2009) indicated that the model is designed having in mind geography and genetics, so it does not consider users identification. An intermediate area may be located between the Inner and the Outer circle, because some people use English just at home, instead of using it for academic, social or political purposes. Another intermediate area may be located between the Outer Circle and the Expanding circle, because there are many countries in which English is not perceived as a Foreign Language anymore, but as a Second Language.

Nelson and Todd (1992) mentioned that in Ghana, English is perceived as a second language and probably as a result of their early exposure to, and intimacy with English, Ghanaians are known, in West Africa, to have developed a particularly positive attitude toward English. At the time when the return to the indigenous languages has virtually become the song of the day in other parts of the continent, Dseagu (1996) reports a sizable minority of middle-class Ghanaians who still give priority to English in their homes, and make their children acquire it as their first language. The attachment of Ghanaians to English, predictably, affects their attitude toward 'New Englishes' in the form of 'Ghanaian English', which is sometimes perceived as Pidgin by educated Ghanaians (Huber, 1998). Ghanaians are also reported to be very proud of their English (Nelson \& Todd 1992:440, Sey(1973:7)

Gyasi (1991: 26), principal in English at the Kumasi branch of the Ghana Institute of Languages, confirms that 'Ghanaians generally boast that their pronunciation is nearer that of RP than that of other nonnative users of English in the former British colonies in Africa, especially in West Africa'. They are also very sensitive to stigmatised forms and will promptly correct themselves if their attention is drawn to a mistake Sey, (1973:7)

This background probably explains why Ghana, despite a shared colonial experience with, and a similar sociolinguistic background to, countries like Nigeria, Cameroon, Sierra Leone, and Gambia, has the tendency to use learned and archaic forms and is also known for flamboyance of prose style and frequent cases 
of hyper-correctness. Little wonder several public speakers, journalists and writers in Ghana who are known for this flamboyant prose style and the use of learned forms are those ascribed linguistic excellence. Confirming this notion are the following expression in Fante: "Brəfo ye dur" (English is weighty, very powerful) "Otu brכfo" (He digs up English) and "Tu bra [Dig it up (An exhortation to public speakers to utter more English expression of pleasing cadences)].

As earlier mentioned, due to the above stated tendency among educated Ghanaians, books, textbooks and other reading materials maybe produced without giving attention to the level of linguistic competence of the targeted audience. Giving credence to this unfortunate situation in Ghana, a recent study by Rubagumya et al. (2010) on Language of Instruction and Quality of Learning in Tanzania and Ghana, findings revealed that in "both Ghana and Tanzania, English textbooks were difficult for learners to read.Language used in most textbooks was above the level learners can understand.

It is pertinent to mention at this juncture that the aforementioned tendency of educated Ghanaians aroused a keen desire in the researcher to conduct in-depth investigation into the readability of University students' handbook of three most reputable Universities in Ghana: University of Ghana, Kwame Nkrumah University of Science and Technology and University of Cape Coast.

\section{Methodology}

In this study, seven readability indexes were applied to sample sections of the university students' handbook in order to ascertain the readability of these handbooks. The study also investigated to ascertain whether there were variations in the readability scores of the seven readability indexes. In answering research question 2 and test the hypothesis, a one-way ANOVA was used at a significant level of 0.05 by means of Statistical Package for Social Sciences (SPSS) version 16.

The readability of the handbooks was determined as shown in Table 2 and 3 using the following readability formulas: Flesch-Reading Ease, Gunning Fog, Flesch-Kincaid Grade Level, Coleman-Liau, SMOG Index, Automated Readability Index and Lisear Write Formula. The seven readability formulas were used based on the recommendation of Burke and Greenberg (2010), that using two or more readability formulas is preferable because the formulas have certain limitations, for example, SMOG readability index often scores higher than other measures while Flesch-Kincaid often scores lower than other measures. When several readability indexes are used, a statistically balanced and accurate result is obtained.

VIII. Results and Findings

Table 2: Readability Consensus Score of Handbooks

\begin{tabular}{|l|l|l|l|}
\hline \multirow{2}{*}{ Institution } & \multicolumn{2}{|l|}{$\begin{array}{l}\text { Readability Consensus on Handbook } \\
\text { (Based on 7 readability formulas in Table 1) }\end{array}$} \\
\cline { 2 - 4 } & Grade Level & Reading Level & Reader's Age \\
\hline University of Ghana, Legon & 16 & Very difficult to read & College Graduate \\
\hline $\begin{array}{l}\text { Kwame Nkrumah University of } \\
\text { Science and Technology }\end{array}$ & 16 & Very difficult to read & College Graduate \\
\hline University of Cape Coast & 19 & Very difficult to read & College Graduate \\
\hline
\end{tabular}

Source: Field Survey, 2013.

The selected portions from each of the students' handbook of the three universities were fed into a computer and subjected to readability test using the seven readability indexes. Various scores obtained coupled with appropriate remarks interpreting the meaning of readability scores are provided in Table 2 above. Inferences drawn based on information in Table 2 and 3 are presented below.

Table 3: Result of Readability Indexes Analysis on University Students' Handbook

\begin{tabular}{|l|l|l|l|l|}
\hline & & & \\
& & & \\
Readability Index & & & & \\
\hline Flesch Reading Ease & 24.7 & 24.6 & 10.7 & Very difficult to read \\
\hline Gunning Fog & 14.8 & 13.4 & 18.6 & Hard to read \\
\hline Flesch-Kincaid Grade Level & 17.2 & 15.7 & 19 & University Graduate and above \\
\hline The Coleman-Liau Index & 12 & 15 & 16 & Twelfth Grade/Graduate College \\
\hline The SMOG Index & 14.1 & 14.7 & 16.1 & University \\
\hline Automated Readability Index & 17.5 & 16.3 & 19.9 & University Graduate \\
\hline Lisear Write Formula & 20.9 & 18.3 & 22.5 & University Graduate and above \\
\hline
\end{tabular}

Source: Field Survey, 2013. 
Findings from the readability consensus of the seven formulas in Table 3 revealed that on a general note, the handbooks of the three universities are very difficult to read. The handbook for the university of Ghana and Kwame Nkrumah University of Science and Technology scored 16 as the overall readability of all the indexes used for the study referred to as readability consensus, whereas University of Cape Coast scored 19, which showed a distinct difference from the other two universities. The results lend support to Rubagumya et al. (2010) and Sey (1973:7) that educated Ghanaians often write with complex grammatical structures to the detriment of the readers, who may have difficulty comprehending or getting the import of the text. Essentially, Table 2 has shown that Ghanaian university students' handbook measured in terms of readability indexes are very difficult to read and comprehend.

Findings from Table 3 revealed that for the Gunning Fog readability, the University of Ghana's handbook scored 14.8, Kwame Nkrumah University of Science and Technology scored 15.7 and University of Cape Coast scored 18.6. Flesch Reading Ease had the following: 24.7; 24.6 and 10.7, the SMOG Index has: 14.1; 14.7 and 16.1 and Lisear Write Formula has: 20.9; 18.3; 22.5. Though, the scores of the readability indexes vary, they all pointed to the fact that the handbooks are very difficult to read and comprehend. For example, Gunning Fog scores for the three handbooks revealed that they are hard to read, the implication of hard to read can be understood in the light of Ivan's (2010) statement that the ideal score for readability with the Fog index is 7 or 8 . Anything above 12 is too hard for most people to read. For instance, The Bible, Shakespeare and Mark Twain have Fog Indexes of around 6. The leading magazines, like Times, Newsweek, and the Wall Street Journal average around 11. This means that the language use and style may be difficult for students to comprehend, left alone act in harmony with the requirement and information in the handbook. Table 3 showed that the handbooks were written beyond students' comprehension level making the handbooks suitable only for University graduates. This is in harmony with the result of Dzinyela et al. (2003) that writers in Ghana often write beyond students' reading level.

In summary, Table 3 has vividly shown that there is variation in the scores of readability indexes, with majority of the indexes showing that the handbooks are very difficult to read and could only be comprehended by university graduates and above.

Table 4: Means and Standard Deviation of Readability Indexes Analysis Scores

\begin{tabular}{|c|c|c|c|c|c|c|c|c|}
\hline & \multirow[b]{2}{*}{$\mathrm{N}$} & \multirow[b]{2}{*}{ Mean } & \multirow[b]{2}{*}{ Std. Deviation } & \multirow[b]{2}{*}{ Std. Error } & \multicolumn{2}{|c|}{$95 \%$ Confidence Interval for Mean } & \multirow[b]{2}{*}{ Minimm } & \multirow[b]{2}{*}{ Maximum } \\
\hline & & & & & Lower Bound & Upper Bound & & \\
\hline UG & 7 & 17.3143 & 4.32721 & 1.63553 & 13.3123 & 21.3163 & 12.00 & 24.70 \\
\hline KNUST & 7 & 16.8571 & 3.73580 & 1.41200 & 13.4021 & 20.3122 & 13.40 & 24.60 \\
\hline UCC & 7 & 17.5429 & 3.34629 & 1.36612 & 13.2049 & 20.2284 & 10.70 & 19.90 \\
\hline Total & 21 & 16.9750 & 3.65180 & .81657 & 15.2659 & 18.6841 & 10.70 & 24.70 \\
\hline
\end{tabular}

Source: Field survey, 2013.

UG: University of Ghana, Legon KNUST: Kwame Nkrumah University of Science and Technology and UCC: University of Cape Coast

Table 4 reveals the mean scores of the various handbooks: University of Ghana $=17.31$, Kwame Nkrumah University of Science and Technology $=16.86$ and University of Cape Coast $=17.54$. The lower and the upper bounds at $95 \%$ confidence interval of the university students' handbook were within the same range and used to measure if there is any significant difference in readability of the mean scores of the three handbooks.

Table 5: One Way ANOVA Showing Differences in the Mean Scores of the Three Handbooks

\begin{tabular}{llllllll}
\hline Readability & \multicolumn{1}{l}{ Sum } & of & & & & \\
\hline & Squares & & df & Mean & Square & F & Sig. \\
\hline Between Groups & 1.707 & 2 & .853 & & .055 & .947 & \\
Within Groups & 280.743 & 18 & 15.597 & & \\
Total & 282.450 & 20 & & & & \\
\hline
\end{tabular}

Source: Field survey, 2013. $P<.05$

One way Analysis of variance (ANOVA) between groups was computed to test whether statistically significant differences exist among the mean scores of the three handbooks of the universities. The results in Table 5 revealed that the readability of Ghanaian university students' handbook does not differ significantly across the three handbooks, $F(2,18)=0.55, \mathrm{p}=.947$. The results provided answer to the third research question 
indicating that there is no significant difference statistically in the readability of Ghanaian university students' handbook.

\section{Hypothesis: Ghanaian university students' handbooks are difficult to comprehend when measured in terms of readability indexes.}

The purpose of this hypothesis was to ascertain the relative ease or level of readability and comprehensibility Ghanaian university students' handbooks offer prospective readers. The information presented in Table 2 and 3 showed that the handbooks are very difficult to read. The readability consensus of seven readability indexes revealed that the content of the handbooks are only suitable for university graduates and those with higher educational qualification. By virtue of the results, readability indexes the researcher fails to reject the null hypothesis: Ghanaian university students' handbooks are difficult to comprehend when measured in terms of readability indexes.

\section{Conclusion and Recommendation}

The investigative and analytical approach adopted for this study have vividly shown that Ghanaian university students' handbooks are very difficult to comprehend when measured in terms of readability indexes. The seven readability indexes used in ascertaining or testing the readability of the three handbooks revealed that they are very difficult to read, with result indicating that they were written to meet the level of university graduates and those with higher educational qualification as depicted in Table 2 . The study also discovered that there are variations in the scores of readability indexes. Table 3 vividly portrays these variations, though the variations are little, results thus show that the handbooks are altogether difficult to read and comprehend.

The result of the One Way ANOVA in Table 5 vividly revealed that there are no significant differences statistically across the three handbooks. The study also failed to reject the hypothesis that Ghanaian university students' handbooks are difficult to comprehend when measured in terms of readability indexes. Tables 2 and 3 lend support to this conclusion.

It is pertinent to state that while readability is only one element in text selection, it is important, and should not be ignored or handled with laxity. Appropriate readability check should be done so as to adequately provide students with useful and functional information. In line with Sey (1973:7), complex grammatical structures and learned forms as well as unnecessary display of flamboyant prose style should be avoided in producing students' handbook. The hallmark of text contained in students' handbook should be sensitive to students' reading level. Attainment of these qualities in the production of students' handbook undoubtedly will go a long way to awaken the dying embers of handbook readability and comprehensibility.

\section{References}

[1]. Boghossian, P.A. (2001). What is social construction? Times literary supplement, 23: 6-8

[2]. Burke, V., and Greenberg, D. (2010). Determining readability: How to select and apply easy-to-Use readability formulas to assess the difficulty of adult literacy materials. Adult Basic Education and Literacy Journal, 4, 34-42.

[3]. Drucker, M. R. (2012). Student handbook. http://www.quinnipiac.edu/student-experience/health-and-safety/student-handbook/. Retrieved August 5, 2013.

[4]. Dzenyela J. M., Opare, J. A., Obeng, E. A. (2003). The role and contribution of higher education to improving the quality of basic education. ADEA, 12 (3), pp. 15-22.

[5]. Flamand, L. (1999). What is social constructionism? Retrieved from www.ehow.com $>$... $>$ Governmentpolitics $>$ socialtheory on January $11,2011$.

[6]. Gergen, K. J. (1985). The social constructionist movement in modern psychology.American psychology association 40, 3 : 266 - 275.

[7]. Gyasi, I. K. (1990). The state of English in Ghana. English Today 23, 24-6.(1991). Aspects of English in Ghana. English Today, pp. 26-31

[8]. Hackling, L. (1999). The social construction of what? Harvard: Harvard University Press.

[9]. Hyland, K. (1999). Academic attribution: Citation and the construction of the disciplinary knowledge. Applied Linguistics, 20.3:341-67

[10]. Ivan, A. (2010). Klariti.com. Fog Index and Readability Formulas. Available at http://www.klariti.com/business-writing/FogIndex-Readability-Formulas.shtm1\#top.. Accessed on March 12, 2013.

[11]. Katerm, K., Devise, I., Flament, I. \& Loots, G. (2004). Two practices, one perspective, many constructs: on the implications of social constructionism. Retrieved from as.nyu.edu/docs/10/1153/socialconstructionism.pdf on 17/4/2010 at 12:30pm.

[12]. Lively, B. A., and Pressey, S. L. (1923). A method of measuring vocabulary burden oftextbooks. Educational Administration and Supervision, 9, 389-398.

[13]. Matz, R. G. (2012). The importance of maps. http://www.umapper.com/blog/?p=2455. Retrieved on August 5, 2013.

[14]. Nelson, C. N., and Todd, L. (1992). Ghana. In McArthur 1992:440.Nordquist, R. (2013). World Englishes. http://grammar.about.com/od/words/ a/Englishasgloballanguage.htm

[15]. Pannekoek, F. (2012). Athabasca Student Handbook. http://www.athabascau. ca/handbook/index.php. Retrieved August $4,2013$.

[16]. Rorty, R. (1979). Philosophy and the mirror of nature. Princeton, NJ: Princeton University Press.

[17]. Rubagumya, C., Kiliku, P., Mapunda, G., Muhdhar, R., Mtana, N., Tarimo, E. Ankomah, Y., Forde, L. D, Osei-Amankwah, L., Afitska, O., Clegg, J. (2010) Language of Instruction and Quality of Learning in Tanzania and Ghana. EdQual Policy Brief No 2, $09 / 10$ p. 1 .

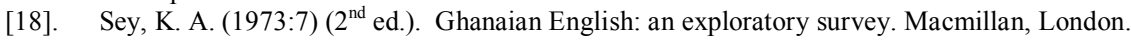


[19]. Vygotsky, L. (1978). Mind in society: the development of higher psychological processes. M. Cole, V. Johnsteiner, S. Scribner, \& E. Souberman (Eds.) (pp. 19-37) Cambridge, MA: Harvard University Press.

[20]. Wellington, J. \& Osborne, J. (2001). Language and literacy and science education. Buckingham, England: Open University Press.

\section{Sample Texts from University of Ghana Students' Handbook}

The campus of the University lies about 13 kilometres north-east of Accra, the capital of Ghana, at an altitude of between 90 and 100 metres. From the Main University Gate on the Dodowa Road, the University Avenue extends to Commonwealth Hall on Legon Hill. Along it are grouped other Halls of Residence, Departments, lecture theatres and laboratories. Mid-way, an open space - the University Square - with an ornamental pool is over-looked by the Balme Library (named after David Mowbray Balme, the first Principal of the University College). Across from the University Square are sports fields, a Central Cafeteria and halls of residence. Behind Commonwealth Hall is an open-air theatre with a Grecian style auditorium build into the slope of Legon Hill. On the summit of Legon Hill is the Convocation Group of Buildings which houses the University's administration offices, The Great Hall, with a seating capacity of 1,500 and a Tower donated by the Government of Ghana in 1959 to commemorate Ghana's Independence. On the southern side of the campus are residential accommodation for staff, the University Basic Schools, the Noguchi Memorial Institute for Medical Research, School of Public Health, Sports Stadium, a night market, supermarket and student hostels; while on the Northern side are more teaching departments, lecture theatres and laboratories, Across the Accra-Dodowa road from the Main University Gate is a Police Station, a university Hospital and housing for Junior Staff of the University.

\section{Result:}

Flesch Reading Ease score: 24.7 (text scale)

Flesch Reading Ease scored your text: very difficult to read.

Gunning Fog: 14.8 (text scale)

Gunning Fog scored your text: hard to read.

Flesch-Kincaid Grade Level: 17.2

Grade level: College Graduate and above.

The Coleman-Liau Index: 12

Grade level: Twelfth Grade

The SMOG Index: 14.1

Grade level: college

Automated Readability Index: 17.5

Grade level: College graduate

Linsear Write Formula : 20.9

Grade level: College Graduate and above.

\section{Sample texts from of Kwame Nkrumah University of Science and Technology Students' Handbook}

The University Hospital is part of the University Health Services. It is under the supervision of the Director, University Health Services, the Hospital is a 24-Hour General Hospital, established and managed by the KNUST. It is located in the Northeastern part of KNUST campus, along the Kumasi-Accra Highway. The Hospital consists of 100 beds and cots, an out-patient Department (OPD), Four Wards, Operation Theatre, Laboratory, X-Ray, Maternity, Maternal and Child Health units, Dispensary, Medical Records Unit, a Dental Clinic and Eye Clinic. The services provided include Out-Patient Services, in-Patient Services, Maternal Care, Radiography, Ultra Sound, Surgery, Laboratory, Dental Care and Ambulance Services, Medical attention is given free of charge to students, members of staff and their registered dependants. Arrangements are usually made for specific cases to receive specialist attention where necessary, outside the university Hospital.

Flesch Reading Ease score: 24.6 (text scale)

Flesch Reading Ease scored your text: very difficult to read.

Gunning Fog: 13.4 (text scale)

Gunning Fog scored your text: hard to read.

Flesch-Kincaid Grade Level: 15.7

Grade level: College Graduate and above.

The Coleman-Liau Index: 15

Grade level: college

The SMOG Index: 14.7

Grade level: college

Automated Readability Index: 16.3

Grade level: College graduate 
Linsear Write Formula : 18.3

Grade level: College Graduate and above.

\section{Sample texts from University of Cape Coast Students' Handbook}

The Vice-Chancellor is the academic and administrative head of the University, and chief disciplinary officer is responsible to the University Council for the overall administration of the University. He has to be informed of all important developments and problems in the University, in particular those relating to academic activities, finance, physical developments and student affairs. Apart from his/her functions within the University, the Vice-Chancellor also serves as spokesperson in relation to the government and external bodies including the international community. In the actual execution of his/her duties, the Vice-Chancellor works mainly through the various Boards/Communities which are responsible for policy-making, some of which he/she serves on as Chairperson. Even when he/she does not serve as Chairperson of some Committees/Boards, the Statues provide that he/she should have unrestricted rights of attendance and speech at all meetings of University bodies and he/she either receives or has access to minutes of all University bodies. The Pro-ViceChancellor, who is assigned special responsibilities by the Statues, acts for the Vice-Chancellor when the latter is absent.

Flesch Reading Ease score: 10.7 (text scale)

Flesch Reading Ease scored your text: very difficult to read.

Gunning Fog: 18.6 (text scale)

Gunning Fog scored your text: difficult to read.

Flesch-Kincaid Grade Level: 19

Grade level: College Graduate and above.

The Coleman-Liau Index: 16

Grade level: graduate college

The SMOG Index: 16.1

Grade level: graduate college

Automated Readability Index: 19.9

Grade level: College graduate

Linsear Write Formula: 22.5

Grade level: College Graduate and above. 